\title{
Uso de las tecnologías de información y comunicación en adolescentes de las instituciones educativas públicas del Distrito Mi Perú
}

\author{
Use of information and communication technology by adolescents of public educational \\ institutions at Mi Peru District
}

\author{
Juan Morales Quispe ${ }^{1,2}$ \\ 1 Docente, Facultad de Ciencias de la Salud, Universidad de Ciencias y Humanidades, Lima, Perú. \\ ${ }^{2}$ Médico cirujano, Dirección Regional de Salud del Callao, Perú.
}

\begin{abstract}
Resumen
Los conocimientos sobre los efectos del uso de las tecnologías de información y comunicación (TIC) en adolescentes aún son insuficientes. Objetivos. Determinar la frecuencia y nivel de consumo de las TIC en estudiantes. Diseño. Estudio transversal. Institución. Tres colegios estatales del distrito Mi Perú, Región Callao, Perú. Participantes. Estudiantes de colegios estatales. Intervenciones. Para medir la frecuencia y nivel de consumo de las TIC se empleó la escala de autoevaluación del Instituto de Adicciones de Madrid Salud, que considera cuatro dimensiones. Principales medidas de resultados. Frecuencia y nivel de consumo de las TIC. Resultados Se encuestó a 459 sujetos entre 14 y 19 años, 226 (49,2\%) varones y $233(50,8 \%)$ mujeres. El 40,3\% tenía internet en casa, 55,1\% accedía a cabinas públicas, 33,3\% las usaba diariamente, 43,1\% 2 a 3 veces/semana, $76,5 \%$ se conectaba de 3 a 5 horas/semana. Los usos más frecuentes fueron: redes sociales ( $87,4 \%)$, descarga de música/video (70,2\%) y buscar información académica $(93,2 \%)$. Conclusiones. El 92,6\% de los adolescentes se clasificó como usuario sin problemática y el nivel de uso de las TIC fue bajo.

Palabras clave. Tecnologías de información y comunicación (TIC); Adolescentes; Callao; Perú.
\end{abstract}

\section{Abstract}

Knowledge on use of information and communication technology (ICT) effects on adolescents is still insufficient. Objectives: To determine ICT frequency and level of use by students. Design: Cross-sectional study. Setting: Three national schools from Mi Peru district, Callao Region, Peru. Participants: National schools students. Interventions: A Madrid Health Addictions Institute autoevaluation scale was used to determine ICT frequency and level of use; it considers four dimensions. Main outcome measures: Frequency and level of use of ICT. Results: Four hundred and fifty-nine 14 to 19 year-old subjects were surveyed, $226(49.2 \%)$ were male and 233 (50.8\%) female; $40.3 \%$ had internet at home, 55.1\% used public cabins, 33.3\% used it daily, $43.1 \% 2-3$ times/week, and $76.5 \% 3-5$ hours/week. Most common uses were social networks (87.4\%), downloading music/video (70.2\%) and obtaining academic information (93.2\%). Conclusions: In the adolescents studied $92.6 \%$ were classified as users without problems. Level of TIC use was low.

Keywords: Technologies of information and communication; Adolescents; Callao; Peru.

\section{An Fac med. 2015;76(3):257-9 / http://dx.doi.org/10.15381/anales.v76i3.11235}

\section{INTRODUCCIÓN}

La literatura señala los riesgos asociados al uso que la población adolescente hace de las tecnologías de información y comunicación (TIC), como los contenidos inapropiados, contactos con desconocidos, amenazas a la privacidad, aislamiento social, dependencia y adicción, sedentarismo, suspensión de actividades anteriormente satisfactorias como los deportes, juegos o actividades en familia ${ }^{(1,2)}$. El uso intensivo de internet (más de 2 horas/día) se relacionan con sobrepeso, sueño insuficiente y puntuación más elevada en las escalas de depresión ${ }^{(3)}$.

Los conocimientos sobre los efectos de las TIC en los adolescentes aún son in- suficientes. Resulta necesario conocer la frecuencia y nivel de consumo de las TIC en los adolescentes, habiéndose realizado un estudio en el Distrito Mi Perú, Región Callao.

\section{MÉTODOS}

Se realizó un estudio descriptivo transversal. Participaron adolescentes que cursaban el cuarto y quinto año de tres colegios estatales del distrito Mi Perú, en la Región Callao. La recolección de datos se realizó entre octubre y diciembre del 2013. La participación de los estudiantes fue voluntaria, contando con el asentimiento y consentimiento informado. Para medir el nivel de uso de las TIC se empleó la escala de autoevaluación del Instituto de Adicciones de Madrid Salud, que considera cuatro dimensiones (chat, navegación por internet, uso de móvil o correo electrónico y uso de las tecnologías del juego), clasificándose en uso sin problemática (SP), con problemática media (PM) y con problemática alta $(\mathrm{PA})^{(4)}$.

\section{RESULTADOS}

Según los criterios del estudio, 459 participantes fueron válidos, 226 (49,2\%) varones y $233(50,8 \%)$ mujeres, con edades entre los 14 y 19 años, con una media de 16,1 años. El procesamiento de datos con la versión 22 del SPSS re- 
sultó en que 33,3\% usaba internet diariamente, $43,1 \% 2$ a 3 veces/semana, $11,3 \%$ una vez/semana. El 76,5\% accedía a internet de 3 a 5 horas/semana, $9,6 \%$ de 6 a 10 horas/semana, $3,9 \%$ de 11 a 15 horas/semana, $2,2 \%$ de 16 a 20 horas/semana y $2,8 \%$ más de 20 horas/ semana. El 0,2\% usaba el chat, $87,4 \%$ redes sociales, $20,9 \%$ correo electrónico, $40,5 \%$ juegos, $70,2 \%$ para descargar música, 93,2\% para buscar información académica. En relación al uso de las TIC, $425(92,6 \%)$ resultaron usuarios SP, 33(7,2\%) con PM y 01 (0,2\%) con PA. En las cuatro dimensiones, el nivel de uso fue bajo, donde el chat y navegación por internet son usados por el $69,5 \%$ y $60,1 \%$ de los adolescentes, respectivamente. El nivel de uso medio predomina en la dimensión navegación por internet y chat, usados en $21,8 \%$ y $17 \%$ de los adolescentes, respectivamente. El nivel alto predomina en las tecnologías del juego, usado en $3,1 \%$ de los adolescentes (tabla 1 y figura 1 ).

\section{DISCUSIÓN}

En nuestro estudio, la proporción de hogares que tuvieron conexión a internet fue inferior respecto a otras latitudes. En España, 85,1\% de los alumnos en Murcia ${ }^{(5)}$ y $88,5 \%$ en Asturias tuvieron internet en el domicilio ${ }^{(6)}$. En VeracruzMéxico, 66,7\% del alumnado poseía computadora y $59,9 \%$ accedía a internet desde el hogar ${ }^{(7)}$. En Barcelona, según el grupo JOITIC (Joves $i$ Tecnologies de la Informació i la Comunicació), 98\% tiene ordenador en casa, $44,8 \%$ lo usa 2 a más horas/día, 98,6\% accede a internet ${ }^{(8)}$.

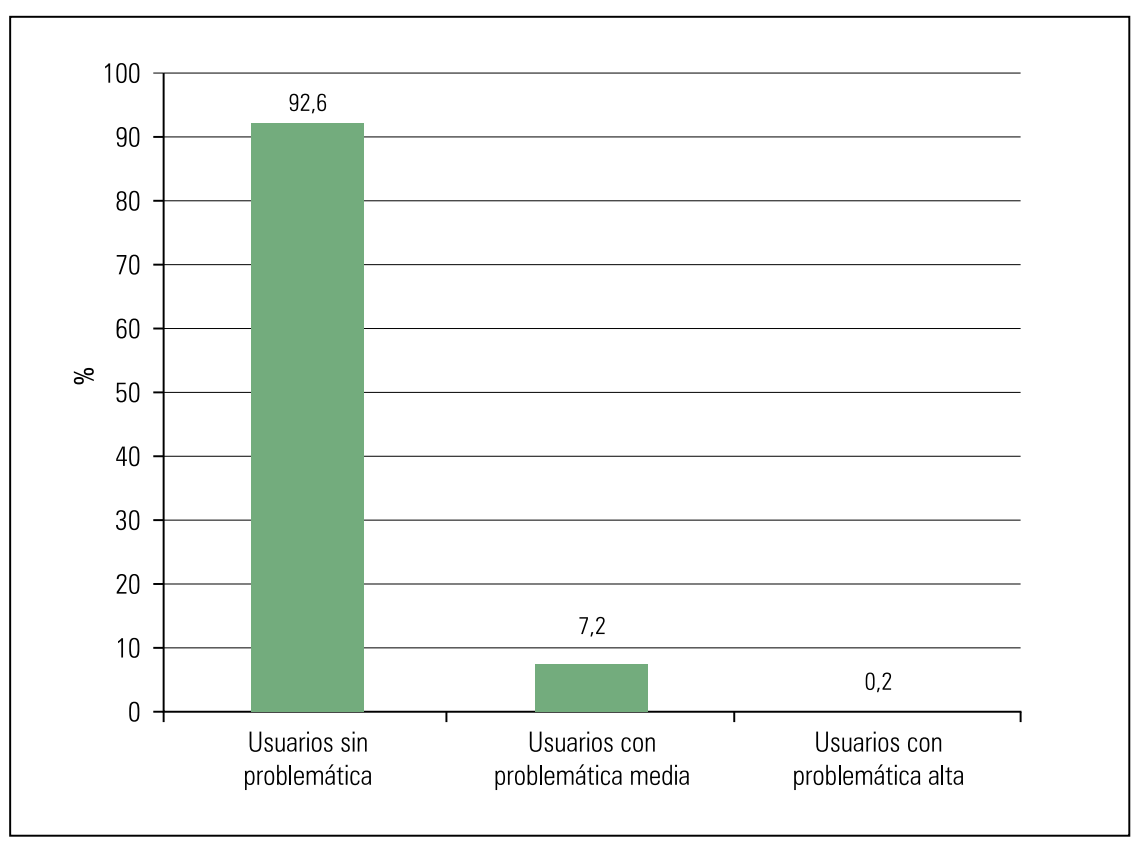

Figura 1. Nivel de uso de las TIC en adolescentes.

Respecto al uso de internet en nuestro estudio, casi la mitad de los participantes la usaba entre 1 y 3 veces/ semana, la mayoría entre 3 y 5 horas/ semana, destacando la búsqueda de información académica, redes sociales y la descarga de música o videos. En España, 60,4\% de los adolescentes accede diariamente a internet, los usos más frecuentes son las redes sociales (85\%), descargar música, imágenes o películas/videos $(64,4 \%)$, buscar información relacionada con los estudios $(60,2 \%)$, correo electrónico $(52,1 \%)$; $28,2 \%$ son usuarios de juegos online ${ }^{(9)}$. En Girona, el 6,7\% de los participantes consume internet más de 30 horas/ semana $^{(10)}$. En Veracruz-México, la internet es usada para comunicarse con amigos o familiares (55\%), bajar música, películas, juegos u otros programas (51,4\%), búsqueda de información para las asignaturas $(50,9 \%)$, y redes sociales $(50,6 \%)^{(7)}$. En Argentina, las prácticas que los adolescentes de los sectores populares y medios realizan con las TIC, la comunicación y el entretenimiento superan ampliamente a las tareas escolares ${ }^{(11)}$. En los adolescentes de Buenos Aires, la red social es el entorno central de entretenimiento y comunicación, con fines de autopresentación, intercambio de contenidos personales entre amistades, búsqueda de relaciones sexo-afectivas y la exploración de distintos aspectos de sociabilidad e identidad $^{(12)}$

Tabla 1. Nivel de uso de la internet según sus dimensiones, en adolescentes de las de las instituciones educativas públicas del Distrito Mi Perú, Región Callao.

\begin{tabular}{|c|c|c|c|c|c|c|c|c|}
\hline \multirow{2}{*}{ Nivel de uso } & \multicolumn{2}{|c|}{ Chat } & \multicolumn{2}{|c|}{ Navegación por internet } & \multicolumn{2}{|c|}{ Móvil o correo electrónico } & \multicolumn{2}{|c|}{ Tecnologías del juego } \\
\hline & $\mathrm{n}$ & $\%$ & $\mathrm{n}$ & $\%$ & $\mathrm{n}$ & $\%$ & $\mathrm{n}$ & $\%$ \\
\hline Baja & 319 & 69,5 & 276 & 60,1 & 266 & 58,0 & 178 & 38,8 \\
\hline Media & 78 & 17,0 & 100 & 21,8 & 55 & 12,0 & 54 & 11,8 \\
\hline Alta & 7 & 1,5 & 7 & 1,5 & 4 & 0,9 & 14 & 3,1 \\
\hline No responde & 55 & 12,0 & 76 & 16,6 & 134 & 29,2 & 213 & 46,4 \\
\hline
\end{tabular}


En nuestro estudio, predomina el uso no problemático de las TIC. El uso problemático se presentó en las tecnologías del juego. Nuestros resultados concuerdan con los estudios del Instituto Madrid, en España, donde la prevalencia del uso problemático de las TIC en los jóvenes madrileños es baja. El 8,5\% de los jóvenes plantea alguna problemática con el uso de los móviles o correo electrónico, reduciéndose a $0,4 \%$ de los jóvenes que reconocen problemas tanto de necesidad, como preocupación o uso compulsivo. En cuanto al uso del chat se reduce al $4,1 \%$ y en las tecnologías del juego plantean una problemática en 3,7\%. Finalmente, la prevalencia de usos problemáticos con la navegación de internet llega a 4,5\% de los participantes ${ }^{(4)}$.

En el Perú, un estudio con mujeres jóvenes de las áreas rurales de Arequipa y Piura, las mujeres usaban las TIC como entretenimiento. El uso de las TIC no se percibe aún como elementos corruptores o riesgosos; mas bien, abre la posibilidad de incrementar el capital social de las mujeres rurales. Las mujeres rurales jóvenes encuentran en las nuevas TIC espacios de privacidad, crecimiento, autoaprendizaje y autorrealización ${ }^{(13)}$. En el Instituto Nacional de Salud Mental de Lima, Cruzado y col reportan que en $83,3 \%$ de los pacientes hospitalizados con diagnóstico de 'adicción a internet', el uso de internet se inició cuando menos dos años antes del ingreso y $50 \%$ se conectaba más de 6 horas/día; el 90\% de pacientes se abocaba a los juegos en red ${ }^{(14)}$.

Sin duda, la internet tiene una infinidad de beneficios en las diversas esferas de la vida. Sin embargo, el uso excesivo, no controlado o supervisado puede llevar a situaciones anormales y comprometer la calidad de vida de los adolescentes.

\section{AGRADECIMIENTOS}

A todos los directores y docentes de las Instituciones Educativas Públicas del Distrito de Mi Perú, de la Región Callao, por haber dado las facilidades para la aplicación del instrumento de recolección de datos. Especialmente a Francisco Flores Jamanca, Víctor Benigno Cárdenas Peña, Luz Ríos Cuadros y Clara Velasco de Leyva.

A Ana María Guerrero Rodas (Lic. en enfermería del Centro de Salud Mi Perú), por haber participado en la aplicación del instrumento de recolección de datos.

A los docentes de la Universidad de Ciencias y Humanidades, por las sugerencias y recomendaciones.

\section{REFERENCIAS BIBLIOGRÁFICAS}

1. Espinar E, López C. Jóvenes y adolescentes ante las nuevas tecnologias: percepción de riesgos. Athenea Digit [Internet]. 2009;16:1-20. Disponible en: http:// www.redalyc.org/articulo.oa?id=53712934004.

2. Garcia-Piña C. Riesgos del uso de internet por niños y adolescentes. Estrategias de seguridad. Acta Pediatr Mex [Internet]. 2008;29(5):273-9. Disponible en: http://www.imbiomed.com.mx/1/1/articulos php? method $=$ showDetail\&id_articulo $=53603 \&$ id_ seccion=3348\&id_ejemplar $=5424$ \&id_revista $=17$.

3. Rivas M, Buñuel J. En adolescentes, el uso excesivo de Internet parece asociarse a problemas de salud. Evid Pediatr [Internet]. 2011;7(38):01-3. Disponible en: http://www.evidenciasenpediatria.es/files/4111240-RUTA/38AVC.pdf.

4. Instituto de Adicciones de Madrid Salud. Estudio de uso problematico de las tecnologias de la informacion, la comunicación y el juego entre los adolescentes y jóvenes de la ciudad de Madrid [Internet]. 2008 p. 01-174. Disponible en: http:// www.madridsalud.es/publicaciones/adicciones/ doctecnicos/UsoProblematicoTIC.pdf

5. Ballestas J, Cerezo C, Veas A. Los jóvenes de educación secundaria ante el uso y consumo de Ias TIC. Rev Cient Electron Educ y Comun en la Soc del Conoc [Internet]. 2014;1(14):22-40. Disponible en: http://www.ugr.es/ sevimeco/revistaeticanet/ numero141/Articulos/Formato/189.pdf.

6. Miguel González I, Echevarria Broz C, Ferrero Fernández E, Suárez Gil P. [Internet use by teenagers from Gijón (Asturias) as a source of health information]. Aten Primaria [Internet]. 2011 Jun [cited 2014 Dec 17];43(6):281-6. Disponible en: http://www. ncbi.nlm.nih.gov/pubmed/20434814.
7. Torres C. Uso de las TIC e internet dentro y fuera del aula. Apertura [Internet]. 2013;13(18):108-19. Disponible en: http://www.redalyc.org/articulo. oa? id=68830443010.

8. Muñoz-Miralles R, Ortega-González R, BatallaMartínez C, López-Morón MR, Manresa JM, Torán-Monserrat $P$. Acceso y uso de nuevas tecnologias entre los jovenes de educacion secundaria, implicaciones en salud. Estudio JOITIC. Aten Primaria [Internet]. 2014 Feb [cited 2014 Nov 19];46(2):77-88. Disponible en: http://www.ncbi. nlm.nih.gov/pubmed/24035765.

9. Rial A, Gómez P, Braña T, Varela J. Actitudes, percepciones y uso de Internet y las redes sociales entre los adolescentes de la comunidad gallega ( España ). An Psicol [Internet]. 2014;30(2):642-55. Disponible en: http://www.redalyc.org/articulo. oa?id=16731188028.

10. Viñas F. Uso autoinformado de Internet en adolescentes: perfil psicológico de un uso elevado de la red. Int J Psychol Psychol Ther [Internet]. 2009;9(1):109-22. Disponible en: http://www. redalyc.org/articulo.oa?id=56012876009.

11. Linne J. Adolescentes de sectores populares: el desafío del estudio en tiempos de entornos digitales. Rev Electron Mutacoes [Internet]. 2014. Disponible en: http://www.academia.edu/7089008/Adolescentes_de_sectores_populares_el_desafio_del_estudio_en_tiempos_de_entornos_digitales.

12. Linne J. Usos comunes de Facebook en adolescentes de distintos sectores sociales en la Ciudad de Buenos Aires. Comunicar [Internet]. 2014;22(43):189-97. Disponible en: http://www. revistacomunicar.com/index.php?contenido $=$ detal les\&numero=43\&articulo=43-2014-19.

13. Garcia A, Barreto M. El uso, apropiación e impacto de las TIC por las mujeres rurales jóvenes en el Perú. Rev Estud para el Desarro Soc la Comun RedesCom [Internet]. 2014;09:251-69. Disponible en: http:// revista-redes.hospedagemdesites.ws/index.php/ revista-redes/article/view/305.

14. Cruzado L, Matos L, Kendall R. Adicción a internet: Perfil clínico y epidemiológico de pacientes hospitalizados en un instituto nacional de salud mental. Rev Med Hered [Internet]. 2006;17(4):196-205. Disponible en: http://www.scielo.org.pe/scielo.php?script=sci_arttext\&pid=S1018$130 \times 2006000400003$.

Artículo recibido el 6 de enero de 2015 y aceptado para publicación el 1 de julio de 2015.

Financiamiento: Autofinanciado.

Conflicto de interés: No existe.

Correspondencia:

Juan Morales Quispe

Dirección: Av Universitaria 5175, Los Olivos, Lima, Perú

Celular: 989521832

Correo electrónico: mdjuanmorales@gmail.com 